\title{
Peritoneal and port-site metastasis following robotic-assisted radical prostatectomy
}

\author{
Ellen O’Connor ${ }^{1}$; Brennan Timm ${ }^{1}$; Bodie Chislett ${ }^{1}$; Jiasian Teh $^{2}$, Nathan Lawrentschuk ${ }^{1}$; \\ Declan G. Murphy'; Damien Bolton ${ }^{1}$ \\ ${ }^{1}$ Department of Surgery, University of Melbourne, Austin Hospital, Heidelberg, Australia; ${ }^{2}$ Division of Cancer \\ Surgery, Peter MacCallum Cancer Centre, Melbourne, Australia
}

Cite as: Can Urol Assoc J 2020 July 17; Epub ahead of print. http://dx.doi.org/10.5489/cuaj.6483

Published online Jul 17, 2020

\section{$* * *$}

\section{Introduction}

Peritoneal metastasis in the absence of further distant metastatic disease is exceedingly rare in prostate cancer. The majority of cases of peritoneal metastases result from widely disseminated metastatic disease, rather than following curative surgical intervention for localized disease. ${ }^{1}$ Large population studies report the incidence of metastasis to the abdominal cavity and peritoneum as less than $1 \%{ }^{2}$ The impact of minimally invasive surgery on oncological outcomes in prostate cancer has been a topic for discussion since its introduction. To our knowledge, there are no dedicated studies looking at robotic-assisted radical prostatectomy (RARP) and its association with peritoneal and port-site metastases. In relation to laparoscopic prostate surgery, there is a reported incidence of port-site metastasis of $0.1 \% .^{3}$

Only eight previous cases reports were identified to demonstrate peritoneal and portsite metastases following RARP. ${ }^{4-10}$ We present a further four cases of peritoneal and portsite metastases following RARP, adding significantly to the published literature in highlighting this unusual occurrence.

\section{Methods}

The records of four cases of peritoneal metastasis following RARP identified across four hospital networks and three surgeons between May 2011 and October 2018 were identified. Patients who had undergone RARP and then subsequently developed peritoneal metastasis without evidence of any further distant metastatic disease were included. Clinical data including presentation, diagnosis, management and outcomes for these patients were obtained. A literature search was conducted using Medline and PubMed databases. 


\section{Results}

\section{Case 1}

A 62-year-old male underwent salvage RARP in June 2018 for Gleason 4+5 prostate adenocarcinoma. This was in response to biochemical prostate specific antigen (PSA) recurrence following primary treatment with external beam radiotherapy (EBRT) seven years earlier. Table 1 outlines initial biochemical and histological characteristics of this and all subsequent cases. In November 2018 he presented with an acute small bowel obstruction. Diagnostic laparoscopy demonstrated extensive peritoneal metastasis and omental caking, biopsy was taken confirming metastatic prostate adenocarcinoma. He underwent an open total omentectomy, ileal resection and end ileostomy and was commenced on androgen deprivation therapy (ADT). Post-operative PSA was initially undetectable however, it has since risen to 0.21 in September 2019 with a small volume of intra-abdominal disease avidity concerning for recurrence on prostate-specific membrane antigen positron emission tomography (PSMA-PET) (Fig. 1).

\section{Case 2}

A 71-year-old male underwent a robotic-assisted cystoprostatectomy in October 2018 for Gleason 3+4 small cell carcinoma of the prostate (Table 1). In January 2019 he developed a subacute large bowel obstruction secondary to a retroperitoneal pelvic mass. Laparotomy was performed revealing significant peritoneal deposits which were debulked and bowel diversion was completed with formation of a sigmoid colostomy. Postoperatively the patient regained a degree of function although due to rapid disease progression he died in February 2019.

\section{Case 3}

A 69-year-old male underwent a RARP for Gleason 4+5 prostate adenocarcinoma in May 2011 (Table 1). He received salvage radiotherapy in August 2013 due to a climbing PSA to $4.49 \mathrm{ng} / \mathrm{mL}$. In January 2019, he was again found to have a slowly rising PSA of $0.54 \mathrm{ng} / \mathrm{mL}$. PSMA PET/CT identified a localized avidity within the anterior abdominal wall (Fig. 2), confirmed by needle biopsy as adenocarcinoma. A laparoscopic-assisted lower abdominal wall resection was performed to remove the well-circumscribed mass consistent with metastatic prostate adenocarcinoma on formal histology. Post operatively PSA remains undetectable.

\section{Case 4}

A 67 -year-old male underwent a RARP for Gleason $4+5=9$ prostate adenocarcinoma with significant ductal component in November 2013 (Table 1). Post-operatively he underwent adjuvant radiotherapy due to histology demonstrating significant multifocal extra-prostatic extension (EPE) and a PSA which remained elevated at $0.8 \mathrm{ng} / \mathrm{mL}$. In June 2014 he presented with worsening abdominal discomfort and a PSA of $445 \mathrm{ng} / \mathrm{mL}$. CT of the abdomen and pelvis revealed peritoneal and omental deposits along with suspected malignant ascites. He 
was treated with androgen deprivation therapy and docetaxel. He eventually died in 2017 due to disseminated disease progression.

\section{Discussion}

Since the implementation of minimally invasive oncologic surgery, the potential for peritoneal and port-site metastases has been an area of concern. Several theories have evolved regarding intraoperative techniques and their contribution to development of peritoneal and port-site metastases. Such theories include the 'seed and soil' hypothesis and the 'chimney effect' whereby turbulent airflow around the port-site whilst establishing pneumoperitoneum may result in implantation of tumour cells. ${ }^{11}$ Additional intra-operative factors such as traumatic tissue removal, tumour morcellation and absence of bag retrieval have been described linking laparoscopic surgery to increased risk of local tumour recurrence. ${ }^{4}$ Emerging concern of peritoneal desiccation and impaired immune response secondary to use of dry $\mathrm{CO} 2$ for insufflation has been the basis of several in vitro and animal studies. Nduka et al ${ }^{12}$ examined this phenomenon in rats and demonstrated an increased rate of peritoneal tumour spread with cold, dry $\mathrm{CO} 2$ insufflation when compared with warmed, humidified $\mathrm{CO} 2$. Few randomized control studies have sought to further investigate these theories to-date, however a definitive association has not yet been established. ${ }^{13}$ Newer devices such as the AirSeal ${ }^{\circledR}$, which aims to reduce lens fogging and decrease smoke accumulation, also introduces higher $\mathrm{CO} 2$ flow. Theoretically this may also impact tumour seeding however further evaluation is required. Of note, regarding our presented cases, AirSeal ${ }^{\circledR}$ was employed in case 2 only.

Many of these theories may additionally relate directly to minimally invasive roboticassisted surgery. Reduced tactile feedback in robotic surgery has been postulated as a limitation to this technique, with suboptimal tissue handling being suggested, although not yet proven to be, a risk factor for peritoneal metastasis. ${ }^{4}$ Although precautionary measures to avoid spillage of malignant cells into the operative field are taken universally, careful handling of high-risk tumours is imperative.

The biological characteristics of the primary tumour is likely to contribute to risk of peritoneal spread. Nguyen et al. examined peritoneal recurrence patterns between open and robotic radical cystectomy for the treatment of muscle invasive bladder cancer and an increased frequency of peritoneal carcinomatosis was found in the robotic cystectomy group $(21 \%)$ when compared to open cystectomy group (9\%). ${ }^{14}$ Over half of cases included in their study were of solitary peritoneal metastases. The authors attributed this finding to a reflection of tumour biology rather than surgical technique; all cases being associated with $\geq \mathrm{T} 3$ staging. Lonnerfors et al. examined port-site recurrence following robotic gynaecological oncology surgeries in 475 women and identified a rate of $1.9 \%(n=9) .{ }^{15}$ This study failed to provide a comparison between robotic and open or laparoscopic techniques however, but ultimately also found an association with high-risk histology. With respect the presented case series, histological patterns of disease with worse prognosis including the presence of small cell and ductal prostate carcinoma is notable. The remaining two cases exhibited Gleason score of 9 , 
further substantiating that peritoneal metastasis seem more predominant in patients with highrisk tumours.

In conclusion, we present a series of peritoneal and post-site metastases following RARP in the absence of more widely disseminated disease. Larger studies are needed to evaluate any association between robotic-assisted surgery and peritoneal metastases. It is essential to explore any potential influences on tumour spread, including the iatrogenic impact from current surgical technique. Tumour characteristics should be considered a likely contributing factor. Modern imaging techniques such as PSMA-PET may enable better diagnosis and guide treatment.

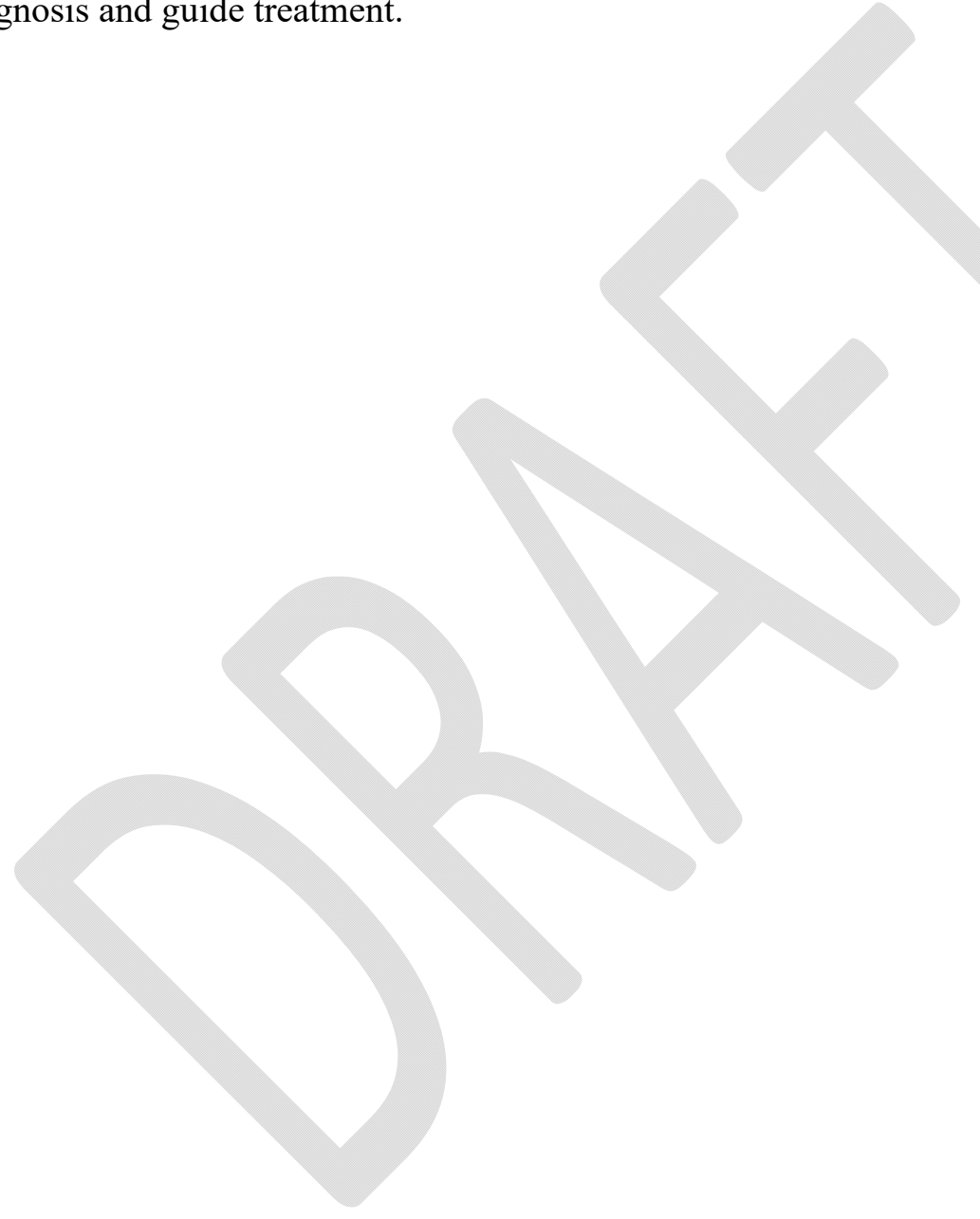




\section{References}

1. Gandaglia G, Abdollah F, Schiffmann J, et al. Distribution of metastatic sites in patients with prostate cancer: A population-based analysis. Prostate. 2013;74(2):210-216. doi:10.1002/pros.22742

2. Riihimaki M, Thomsen H, Sundquist K, et al. Clinical landscape of cancer metastases. Cancer Med. 2018;7(11):5534-5542. doi:10.1002/cam4.1697

3. Tsivian A, Sidi AA. Port site metastases in urological laparoscopic surgery. J Urol. 2003;169(4):1213-1218. doi:10.1097/01.ju.0000035910.75480.4b

4. Acar O, Esen T, Bavbek S, et al. Port site and peritoneal metastases after robotassisted radical prostatectomy. Int J Surg Case Rep. 2014;5(3):131-134. doi:10.1016/j.ijscr.2014.01.010

5. Baber J, Mclaughlin T, Wagner J. Two Unique Cases of Peritoneal Carcinomatosis Following Robotic Assisted Radical Prostatectomy Robot. J Urol Surg. 2019;6(2):152-155. doi:10.4274/jus.galenos.2018.2446

6. Bruyne P, Schatteman P, Naeyer G, et al. Original Port site metastasis in prostate cancer. Can Urol Assoc J. 2015;9(5-6):387-389.

7. Jundt MC, Broski SM. Prostate cancer laparoscopic port site metastasis. Medicine (Baltimore). 2018;91(51):e13757.

8. Sheng J, Findley TW, Sadeghi-nejad H. Urology Case Reports Isolated Non-ascitic Peritoneal Carcinomatosis from Metastatic Prostate Cancer. Urol Case Reports. 2017;10:14-15. doi:10.1016/j.eucr.2016.10.004

9. Shin YS, Doo AR, Kim MK, et al. First case of peritoneal seeding of prostate cancer during robot-assisted laparoscopic radical prostatectomy. Can J Urol. 2012;19(3):6303-6305.

10. Calderoni L, Deliu VM, Farol A, et al. Single Subcutaneous Prostate Cancer Metastasis Detected by 68 Ga-PSMA PET / CT During Early Biochemical Relapse : A Case Report. 2018:1-4. doi:10.1016/j.clgc.2018.11.012

11. Langley RR, Fidler IJ. The seed and soil hypothesis revisited - the role of tumorstroma interactions in metastasis to different organs. 2012;128(11):2527-2535. doi:10.1002/ijc.26031.The

12. Nduka CC, Puttick $M$, Coates $P$, et al. Intraperitoneal hypothermia during surgery enhances postoperative tumor growth. 2002:611-615. doi:10.1007/s00464-0019055-0

13. Matsuzaki S, Jardon K, Maleysson E, et al. Impact of intraperitoneal pressure of a $\mathrm{CO} 2$ pneumoperitoneum on the surgical peritoneal environment $\dagger$. Hum Reprod. 2012;27(6):1613-1623. doi:10.1093/humrep/des081

14. Nguyen DP, Al Hussein Al Awamlh B, et al. Recurrence Patterns After Open and Robot-assisted Radical Cystectomy for Bladder Cancer. Eur Urol. 2015;68(3):399405. doi:10.1016/j.eururo.2015.02.003

15. Lonnerfors CL, Bossmar T, Persson J. Port-site metastases following robot-assisted laparoscopic surgery for gynecological malignancies. 2013;92:1361-1368. doi:10.1111/aogs.12245 


\section{Figures and Tables}

Fig. 1. Prostate-specific membrane antigen positron emission tomography for Case 1 demonstrating pathological uptake in left lower abdomen.

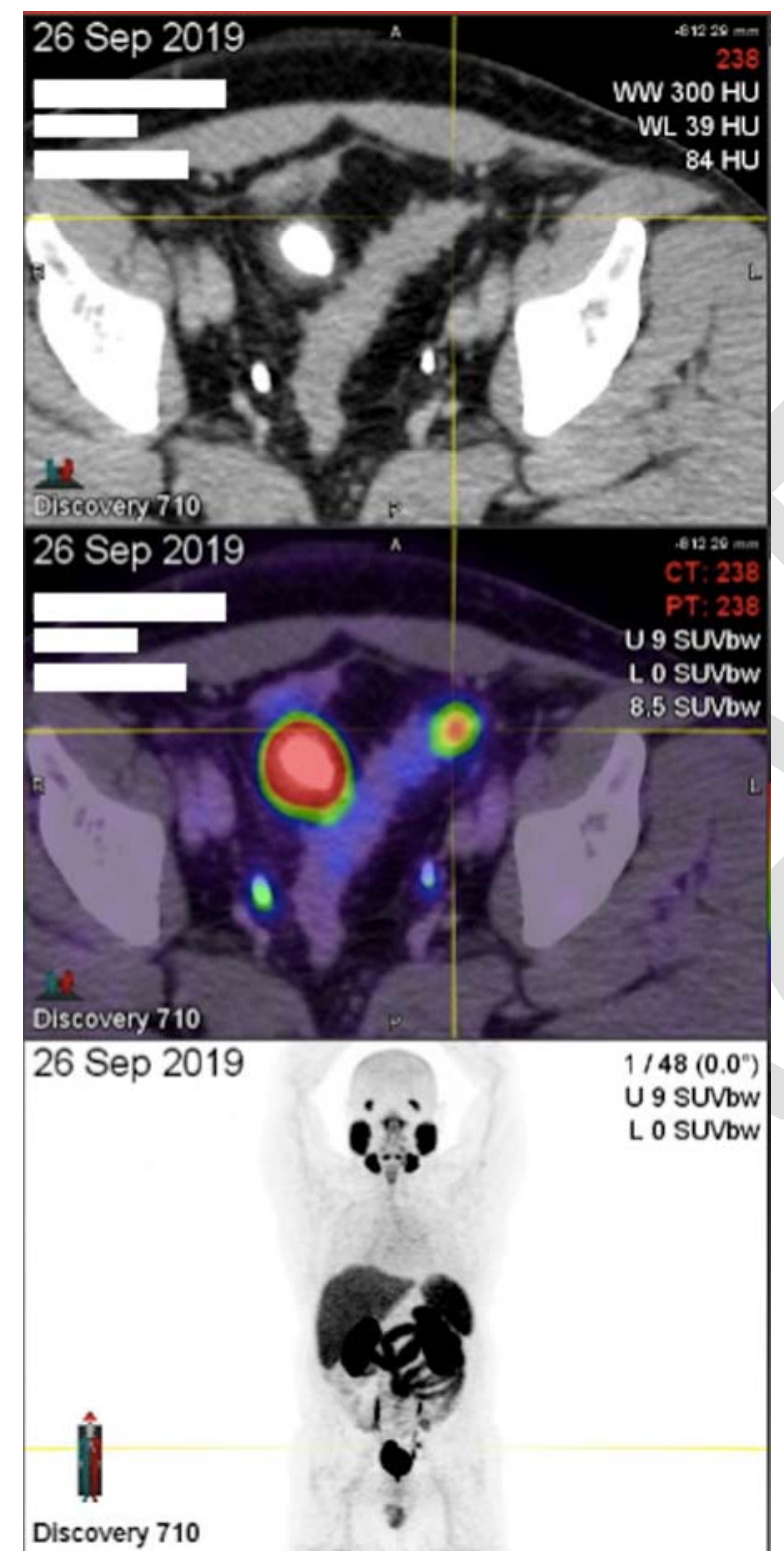


Fig. 2. Prostate-specific membrane antigen positron emission tomography/computed tomography for Case 3 demonstrating pathological uptake in subcutaneous lesion in the midline.

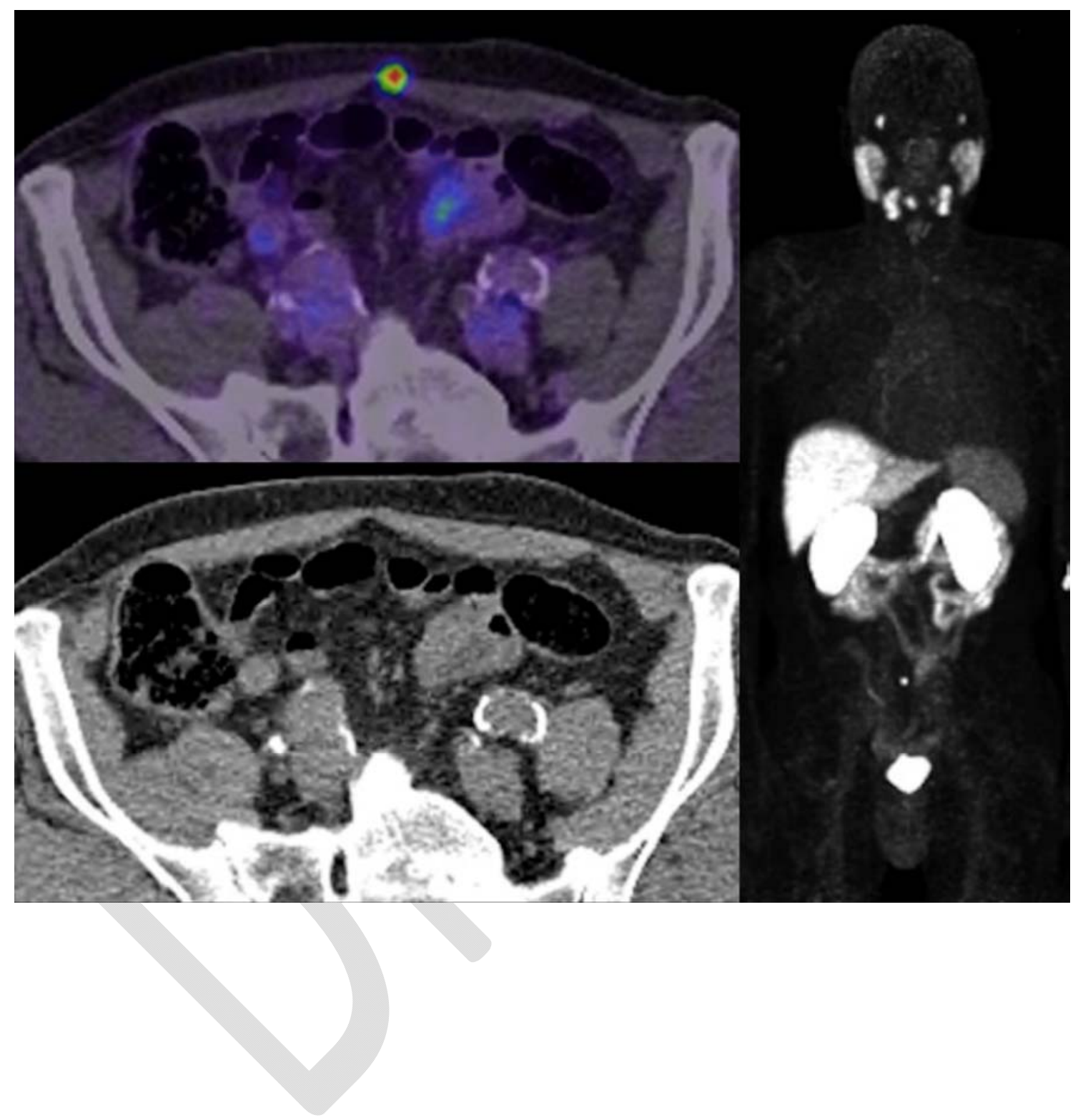




\begin{tabular}{|l|c|c|c|c|}
\hline \multicolumn{6}{|l|}{ Table 1. Biochemical and histological characteristics of case series } \\
\hline Patient & Case 1 & Case 2 & Case 3 & Case 4 \\
\hline Initial PSA & 4.2 & 5.6 & 13.13 & 12 \\
\hline Gleason score & $5+4=9$ & $3+4=7$ & $4+5=9$ & $4+5=9$ \\
\hline TNM & T3bN0M0 & pT4N0M0 & T3aN0M0 & T3aN0M0 \\
\hline Positive margin & $\mathrm{Y}$ & $\mathrm{Y}$ & $\mathrm{Y}$ & $\mathrm{Y}$ \\
\hline EPE & $\mathrm{Y}$ & $\mathrm{Y}$ & $\mathrm{Y}$ & $\mathrm{Y}$ \\
\hline $\begin{array}{l}\text { Seminal vesicle } \\
\text { involvement }\end{array}$ & $\mathrm{Y}$ & Not identified & $\mathrm{N}$ & $\mathrm{N}$ \\
\hline LVI & $\mathrm{N}$ & $\mathrm{Y}$ & $\mathrm{Y}$ & $\mathrm{N}$ \\
\hline PNI & $\mathrm{Y}$ & $\mathrm{Y}$ & $\mathrm{Y}$ & $\mathrm{Y}$ \\
\hline $\begin{array}{l}\text { Histology } \\
\text { Adenocarcinoma }\end{array}$ & $\begin{array}{c}\text { Small cell } \\
\text { carcinoma }\end{array}$ & $\begin{array}{c}\text { Adenocarcinoma, } \\
\text { some regions of } \\
\text { intra-ductal }\end{array}$ & Ductal \\
\hline $\begin{array}{l}\text { Post-RARP } \\
\text { PSA nadir }\end{array}$ & 1.25 & Unknown & $<0.05$ & 0.81 \\
\hline $\begin{array}{l}\text { PSA at } \\
\text { recurrence }\end{array}$ & 6.1 & Unknown & 0.54 & 445 \\
\hline Current PSA & 0.10 & N/A & $<0.03$ & N/A \\
\hline
\end{tabular}

LVI: lymphovascular invasion; PNI: perineural invasion; PSA: prostate-specific antigen; RARP: robotic-assisted radical prostatectomy. 


\begin{tabular}{|c|c|c|c|c|c|c|c|c|c|c|}
\hline Author & Age & $\begin{array}{c}\text { Initial } \\
\text { PSA }\end{array}$ & $\begin{array}{l}\text { Gleason } \\
\text { score }\end{array}$ & TNM & $\begin{array}{c}\text { Initial } \\
\text { treatment }\end{array}$ & $\begin{array}{c}\text { Interval to } \\
\text { metastases } \\
\text { (months) }\end{array}$ & Location & $\begin{array}{c}\text { PSA at } \\
\text { recurrence }\end{array}$ & $\begin{array}{l}\text { Treatment of } \\
\text { recurrence }\end{array}$ & Outcome \\
\hline Acar et $\mathrm{al}^{4}$ & 66 & 6.8 & $4+5$ & $\begin{array}{c}\text { T3aN0M0 + } \\
\text { margin }\end{array}$ & RARP & 21 & $\begin{array}{l}\text { Right hand } \\
\text { port site }\end{array}$ & 0.67 & $\begin{array}{c}\text { Continued } \\
\text { ADT, surgical } \\
\text { resection, } \\
\text { abiraterone, } \\
\text { scheduled } \\
\text { chemotherapy }\end{array}$ & $\begin{array}{l}\text { Rising PSA, further } \\
\text { peritoneal } \\
\text { carcinomatosis at } 7 \\
\text { months, ongoing } \\
\text { chemotherapy }\end{array}$ \\
\hline${\text { Baber et } a l^{5}}^{5}$ & 65 & 4.4 & $3+4$ & T2cN0M0 & RARP & 132 & $\begin{array}{c}\text { Widespread } \\
\text { peritoneal } \\
\text { carcinomatosis }\end{array}$ & 6.6 & $\begin{array}{l}\text { ADT and } \\
\text { abiraterone }\end{array}$ & $\begin{array}{c}\text { Commencing } \\
\text { chemotherapy at } 3 \\
\text { months }\end{array}$ \\
\hline Baber et al $^{5}$ & 65 & 2.7 & $4+5$ & T3bN0M0 & RARP & 24 & $\begin{array}{l}\text { Mesenteric and } \\
\text { pulmonary } \\
\text { nodules }\end{array}$ & 93.9 & $\begin{array}{c}\text { ADT, } \\
\text { docetaxel, } \\
\text { mitoxantrone, } \\
\text { cabazitaxel }\end{array}$ & $\begin{array}{c}\text { Transitioned to } \\
\text { palliative care at } 6 \\
\text { months }\end{array}$ \\
\hline $\begin{array}{l}\text { Bruyne et } \\
\mathrm{al}^{6}\end{array}$ & 46 & 32.8 & $4+3$ & T3bN0M0 & $\begin{array}{c}\text { RARP + } \\
\text { salvage RTx }\end{array}$ & 54 & $\begin{array}{c}\text { Left flank port- } \\
\text { site + Inguinal } \\
\text { lymph node }\end{array}$ & 10.15 & $\begin{array}{l}\text { Surgical } \\
\text { resection }\end{array}$ & PSA 0.09 at 1 month \\
\hline Jundt et $\mathrm{al}^{7}$ & 57 & $\begin{array}{l}\text { Unkno } \\
\text { wn }\end{array}$ & $4+3$ & T2aN0M0 & $\begin{array}{c}\text { RARP + } \\
\text { salvage RTx }\end{array}$ & 60 & $\begin{array}{l}\text { Right rectus } \\
\text { abdominis } \\
\text { muscle }\end{array}$ & 1.5 & $\begin{array}{c}\text { ADT and } \\
\text { chemotherapy }\end{array}$ & $\begin{array}{c}\text { Undetectable PSA } \\
\text { following } 6 \text { cycles } \\
\text { chemotherapy }\end{array}$ \\
\hline
\end{tabular}


CUAJ - Case Series

O'Connor et al

Peritoneal and port-site metastasis after RARP

\begin{tabular}{|c|c|c|c|c|c|c|c|c|c|c|}
\hline Sheng et al ${ }^{8}$ & 60 & 9.5 & $3+4$ & Unknown & $\begin{array}{c}\text { RARP }+ \\
\text { salvage RTx }\end{array}$ & 29 & Omentum & 11.8 & $\begin{array}{l}\text { ADT, surgical } \\
\text { resection, } \\
\text { abiraterone }\end{array}$ & $\begin{array}{l}\text { Peritoneal and lymph } \\
\text { node recurrence } \\
\text { following resection, } \\
\text { ongoing } \\
\text { chermotherapy. }\end{array}$ \\
\hline Shin et $\mathrm{al}^{9}$ & 75 & 10.5 & $4+3$ & $\begin{array}{c}\text { T3aN0M0 }+ \\
\text { margin }\end{array}$ & RARP & 24 & $\begin{array}{c}\text { Liver and } \\
\text { peritoneum }\end{array}$ & 12.37 & $\begin{array}{l}\text { Surgical } \\
\text { resection }\end{array}$ & Unknown \\
\hline $\begin{array}{l}\text { Calderoni et } \\
\mathrm{al}^{10}\end{array}$ & 67 & 8.2 & $4+3$ & pT2c & $\begin{array}{c}\text { RARP + } \\
\text { salvage RTx }\end{array}$ & 37 & $\begin{array}{c}\text { Left-hand port } \\
\text { site }\end{array}$ & 0.98 & $\begin{array}{l}\text { Surgical } \\
\text { resection }\end{array}$ & PSA 0.19 at 1 month \\
\hline Case 1 & 62 & 4.2 & $5+4$ & $\begin{array}{l}\text { T3bN0M0 }+ \\
\text { margin }\end{array}$ & $\begin{array}{l}\text { EBRT + } \\
\text { salvage } \\
\text { RARP }\end{array}$ & 5 & $\begin{array}{l}\text { Omentum and } \\
\text { terminal ileum }\end{array}$ & 6.1 & $\begin{array}{l}\text { Surgical } \\
\text { resection }\end{array}$ & $\begin{array}{l}\text { PSA } 0.10 \text { at } 10 \\
\text { months on ADT }\end{array}$ \\
\hline Case 2 & 71 & 5.6 & $3+4$ & $\begin{array}{l}\text { T4N0M0 + } \\
\text { margin }\end{array}$ & $\begin{array}{c}\text { Robotic } \\
\text { cysto- } \\
\text { prostatectom } \\
\text { y }\end{array}$ & 3 & Peritoneum & Unknown & $\begin{array}{l}\text { Surgical } \\
\text { resection }\end{array}$ & $\begin{array}{c}\text { Deceased at } 3 \text { months } \\
\text { from disease } \\
\text { progression }\end{array}$ \\
\hline Case 3 & 69 & 13.13 & $4+5$ & $\begin{array}{c}\text { T3aN0M0 + } \\
\text { margin }\end{array}$ & $\begin{array}{c}\text { RARP + } \\
\text { salvage RTx }\end{array}$ & 96 & $\begin{array}{l}\text { Midline port } \\
\text { site }\end{array}$ & 0.54 & $\begin{array}{l}\text { Surgical } \\
\text { resection }\end{array}$ & $\begin{array}{c}\text { Undetectable PSA at } 6 \\
\text { months }\end{array}$ \\
\hline Case 4 & 67 & 12 & $4+5$ & $\begin{array}{c}\text { T3aN0M0 }+ \\
\text { margin }\end{array}$ & $\begin{array}{c}\text { RARP + } \\
\text { salvage RTx }\end{array}$ & 7 & $\begin{array}{l}\text { Peritoneum } \\
\text { and omentum }\end{array}$ & 445 & $\begin{array}{c}\text { ADT and } \\
\text { chemotherapy }\end{array}$ & $\begin{array}{l}\text { Deceased at } 3 \text { years } \\
\text { from disease } \\
\text { progression }\end{array}$ \\
\hline
\end{tabular}

ADT: androgen deprivation therapy; EBRT: external beam radiotherapy; PSA: prostate-specific antigen; RARP: robotic-assisted radical prostatectomy. 